\title{
Discriminating secondary from primary non-Gaussian signals
}

\section{P. Abrial,Jean-Luc Starck*,Y. Moudden}

CEA-SAP DAPNIA/SEDI-SAP, Service d'Astrophysique 91191 Gif-sur-Yvette France

E-mail: pabrial@cea.fr, jstarck@cea.fr, ymoudden@cea.fr

\section{N. Aghanim, O. Forni}

IAS-CNRS, Université Paris Sud,Batiment 121,91405 Orsay Cedex, France

E-mail: Nabila.Aghanimeias.u-psud.fr, forni@ias.u-psud.fr

\begin{abstract}
One of the goals in cosmology is to understand the formation and evolution of the structures resulting from the growth of initial density perturbations. Recent Cosmic Microwave Background (CMB) observations indicate that the latter essentially came out of Gaussian distributed quantum fluctuations in the inflationary scenario. However, topological defects (e.g. cosmic strings) could contribute to the signal. One of their important footprint would be the predicted non-Gaussian distribution of the temperature anisotropies. In addition, other sources of non-Gaussian signatures do contribute to the signal, in particular the Sunyaev-Zel'dovich effect of galaxy clusters[01]. In this general context and motivated by the PLANCK experiment, we address the question of finding and discriminating between the different non-Gaussian signatures using both wavelet and curvelet transforms on the sphere to analyse simulated data.
\end{abstract}

$C M B$ and Physics of the Early Universe

20-22 April 2006

Ischia, Italy

\footnotetext{
*Speaker.
} 


\section{Introduction}

This paper describe an application of multiscale methods to feature and non gaussianity detection in spherical map. In this study we use a spherical data analysis software package called MRS available for download at (http://jstarck.free.fr/mrs.html). It works with HEALPIX ${ }^{1}$ and GLESP ${ }^{2}$ pixelization schemes.

\section{Wavelet and Curvelet transform on the sphere}

There are many different possible implementations of a wavelet transform on the sphere and their performance depends on the application. Vielva et al. [05] have developed a mexican hat wavelet on the sphere which they used to detect non gaussianity in full-sky CMB maps. We consider here instead an undecimated isotropic transform which has very similar properties as the $\grave{a}$ trous wavelet tranform. A detailed description of our implementation is given in [02] as well as several applications in denoising and data restoration. Figure 1 shows some back projections of wavelet coefficients at different scales and locations on the sphere.

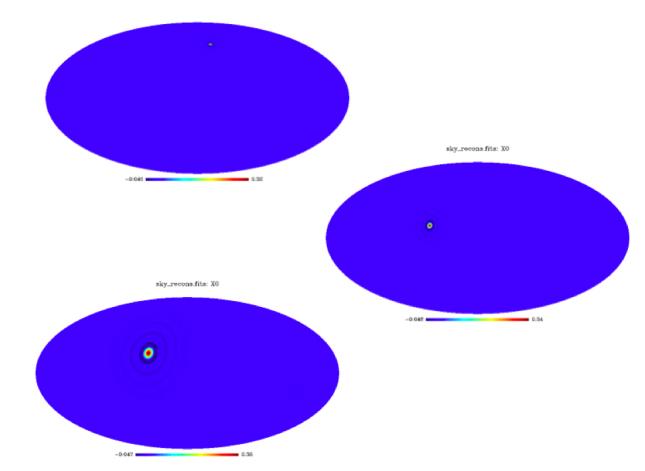

Figure 1: Backprojection of a wavelet coeficients at different scale.

The 2D Curvelet transform introduced by Donoho and Duncan in 2000 in [06] and Starck et al. [04] allows a directionnal analysis of images in different scales. This transform was extended to work with spherical data as fully described in $[02,03]$. The Curvelet transform on the sphere is the result of first applying the isotropic wavelet transform on the sphere, then partitionning the spherical maps at different scales into blocks of appropriate sizes in the Healpix representation, and finally analyzing each block using the usual discrete ridgelet tranform.

\section{Study of non gaussianity}

The 2D curvelet transform was used successfully in [01] along with wavelets to detect and discriminate between different sources non-Gaussianity in flat CMB data maps. As a step towards extending these results to the sphere, we describe a simple experiment in which the multiscale tools presented above are used to detect morphologically different features hidden within Gaussian noise on the sphere.

\footnotetext{
${ }^{1}$ http://healpix.gpl.nasa.gov

${ }^{2}$ http://www.glesp.nbi.dk
} 


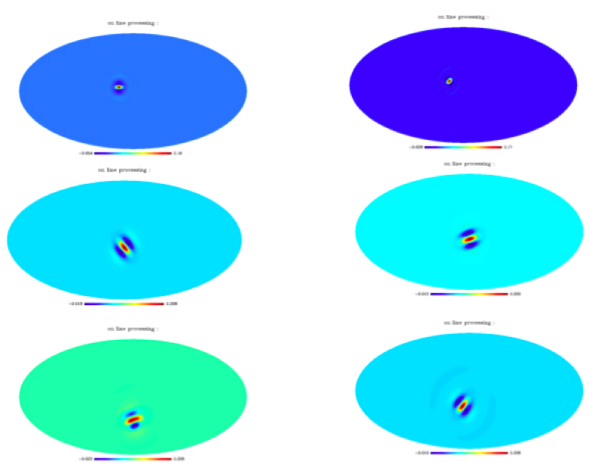

Figure 2: Backprojection of some curvelet coeficients at different scales and locations.

\subsection{Synthetic data}

For this toy experiment, we considered a map with randomly scattered Gaussians to mimic a distribution of SZ Galaxy clusters and a similar map consisting of line segments which mimic cosmic strings. These are shown on figure 3 . We then created a set of simulated images ( $c f$ figure 4 by adding Gaussian white noise with different standard deviations to the two previous images. The Signal to Noise Ratio (SNR) was varied between 0 and 1. The SNR is defined as the pixel values along the lines divided by the noise standard deviation for the linear features, and as the maximum of the Gaussians divided by the noise standard deviation in the case of the Gaussian features.
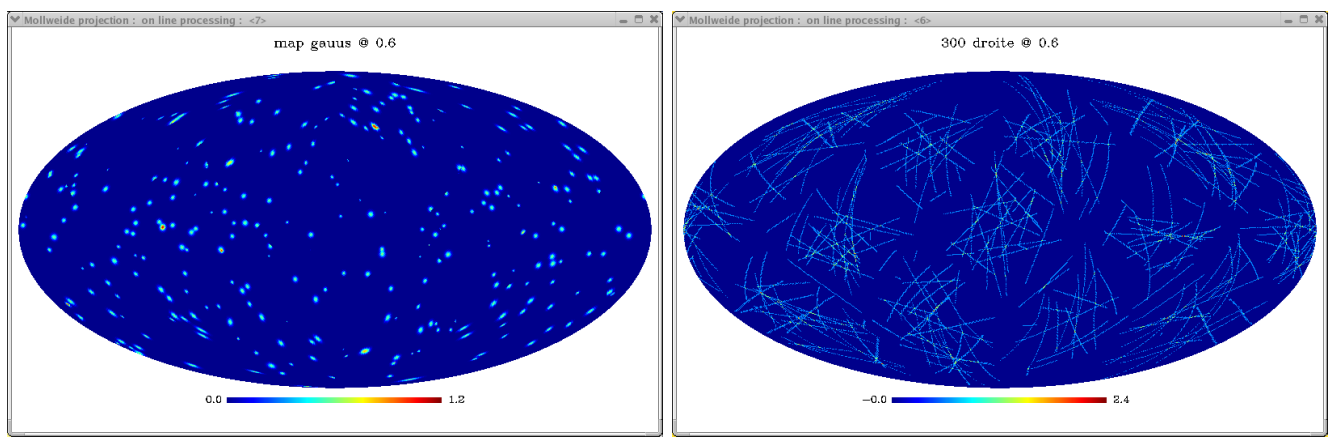

Figure 3: Left : map of gaussian features, Right : map linear features.

\section{Result and conclusion}

On the different wavelet and curvelet scales we compute the kurtosis of the coefficients, normalized by the standard deviation of the kurtosis obtained from the wavelet and the curvelet transform of realizations of Gaussian white noise. Finally we keep the maximum normalized kurtosis along the scales for each SNR value. The results obtained using the wavelet transform (resp. the curvelet transform) on the two data sets are shown on the left (resp right ) of figure 5. The detection power of the wavelet transform is much appears much larger than that of the curvelet transform in the case of isotropic features, while curvelets are more powerful than wavelets in detecting anisotropic features. 

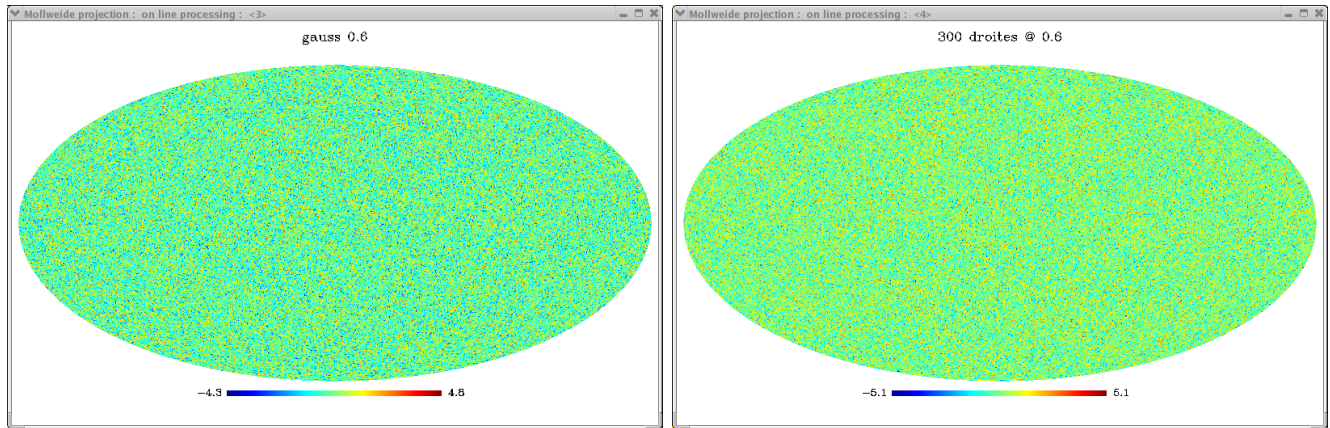

Figure 4: Left : map of gaussian features hidden in gaussian noise, Right : map linear features hidden in gaussian noise.
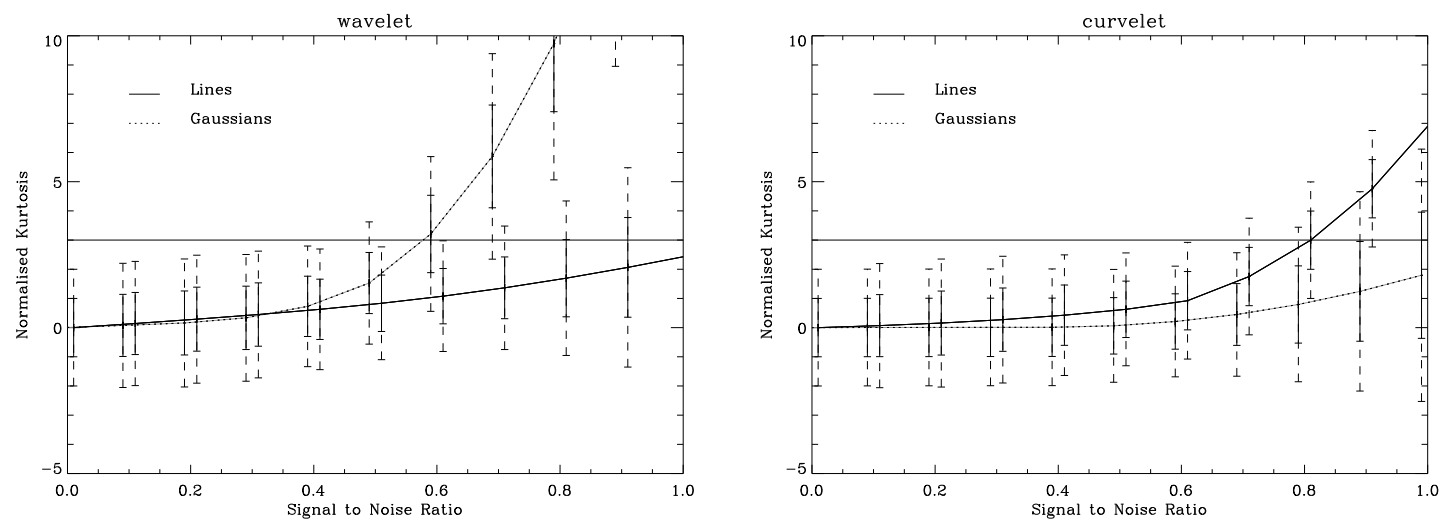

Figure 5: Kurtosis versus the SNR for the two images using the wavelets (left) and the curvelet (right). Dashed lines represent the images with Gaussians and continuous lines represent the images with lines. Continuous error bars correspond to a one sigma level and dashed error bars correspond to a two sigma level.

\section{References}

[01] J.-L. Starck, N. Aghanim and O. Forni, "Detection and discrimination of cosmological non-Gaussian signatures by multi-scale methods", Astronomy and Astrophysics, 416, 9-17, 2004.

[02] J.-L Starck, Y. Moudden, P. Abrial and M. Nguyen, "Wavelets, Ridgelets and Curvelets on the Sphere", Astronomy and Astrophysics, 446, 1191-1204, 2006.

[03] , P. Abrial, J.-L. Starck, Y. Moudden, M.K. Nguyen, "Curvelet Transform on the sphere", IEEE International Conference in Image Processing (ICIP'05) - Sept. 2005.

[04] J.L. Starck, E. Candes, and D.L. Donoho, "The Curvelet Transform for Image Denoising", IEEE Transactions on Image Processing , 11,6, pp 670 -684, 2002.

[05] M.Cruz, E.Martinez-Gonzalez, P.Vielva, L.Cayon, "Detection of a non-Gaussian Spot in WMAP",Mon.Not.Roy.Astron.Soc. , 356 (2005) 29-40.

[06] D.L. Donoho, M.R. Duncan, "Digital curvelet transform: strategy,implementation and experiments", inProc. Aerosense2000, Wavelet Applications VII. 